\title{
Hakim-Adams syndrome secondary to syphilis
}

\author{
Ricardo Krause Martinez de Souza ${ }^{1}$, Alexandre Zatera ${ }^{2}$, Fernanda Leal Suzano ${ }^{2 *}$, \\ Flávia de Oliveira Braga², Samanta Fabrício Blattes da Rocha ${ }^{2}$, Ricardo Ramina ${ }^{3}$, \\ Pedro André Kowacs ${ }^{2}$

\footnotetext{
${ }^{1}$ Ambulatório de Desordens da Memória e do Comportamento, Instituto de Neurologia de Curitiba, Curitiba, Brazil

${ }^{2}$ Instituto de Psiquiatria do Paraná, Curitiba, Brazil; ${ }^{*}$ Corresponding Author: fernandasuzano@uol.com.br

${ }^{3}$ Serviço de Neurologia e Neurocirurgia, Curitiba, Brazil
}

Received 8 May 2013; revised 12 June 2013; accepted 8 July 2013

Copyright (C) 2013 Ricardo Krause Martinez de Souza et al. This is an open access article distributed under the Creative Commons Attribution License, which permits unrestricted use, distribution, and reproduction in any medium, provided the original work is properly cited.

\begin{abstract}
Normal pressure hydrocephalus (NPH) secondary to syphilis is an atypical form of neurosyphilis with an unusual clinical presentation and uncommon neuroimaging findings. A case of Syndrome de Hakim-Adams occurring in a 79year-old male was admitted for investigation of a dementia syndrome. The patient presented with clinical features and neuroimaging compatible with NPH. Syphilis serology was reactive and compatible with active disease. The patient was treated with crystalline penicillin (4 million units every 4 hours for 21 days) and was assessed again after three months, when his cognition and gait had improved and the urinary incontinence had resolved. According to this information, we describe a case of syphilis mimicking normal pressure hydrocephalus, making clear the importance of the investigation of sexually transmitted diseases in the differential diagnosis of dementia, even in patients without established risk supposedly.
\end{abstract}

Keywords: Neurosyphilis; Hakim-Adams Syndrome; Syphilis; Normal Pressure Hydrocephalus

\section{INTRODUCTION}

Syphilis is a sexually transmitted disease caused by the spirochete Treponema pallidum (T. pallidum), which causes neurosyphilis when it invades the nervous system. The initial neurological manifestations of neurosyphilis are asymptomatic meningitis, acute syphilitic meningitis and meningovascular syphilis. In the late stages of the disease, there may be involvement of the spinal cord and cerebral cortex, causing tabesdorsalis or general paresis, which is also known as paralytic dementia [1]. Other presentations of neurosyphilis have been described, such as limbic encephalitis [2] and normal pressure hydrocephalus [3], which are referred to as atypical forms of neurosyphilis.

The aim of this paper is to describe a case of atypical neurosyphilis mimicking NPH in which treatment with crystalline penicillin resulted in a considerable improvement in symptoms.

\section{CASE REPORT}

A 79-year-old male was admitted to the Curitiba Institute of Neurology for investigation of a dementia syndrome. The patient presented with symptoms marked by gait apraxia, dementia and urinary incontinence that had been worsening progressively over 1.5 years. On admission he was taking donepezil $5 \mathrm{mg}$. In the previous six months his symptoms had worsened, leading him to seek a second opinion. Laboratory tests were carried out and the patient had a brain MRI scan, lumbar puncture and neuropsychological assessment.

HIV serology was nonreactive and vitamin B12, folic acid and TSH levels were normal. Syphilis serology was reactive and compatible with active disease (second line of the Table 1).

Cerebrospinal fluid (CSF) analysis revealed an increase in protein concentration $(72 \mathrm{mg} / \mathrm{dL})$ any cellular alterations. Opening pressure was $12 \mathrm{~cm} \mathrm{H}_{2} \mathrm{O}$, and VDRL (venereal disease research laboratory) and FTA-ABS (fluorescent treponemal antibody-absorption) tests were nonreactive.

The MRI revealed ventricular dilation (Evans' index of 0.31 ) out of proportion to the degree of atrophy (Figure 1). Neuropsychological assessment showed cognitive alterations associated with frontal-subcortical dysfunction, confirming the diagnosis of dementia. 
Table 1. Serological profile of syphilis (total antibodies).

\begin{tabular}{cccc}
\hline & CMIA/EIA & RPR & TPHA/TP-PA \\
\hline Non-reactivesyphilis & - & & \\
Active syphilis & + & + & \\
$\begin{array}{c}\text { Treatedorlatentsyphilis } \\
\begin{array}{c}\text { Recent primary syphilis or } \\
\text { false positive }\end{array}\end{array}$ & + & - & + \\
\hline
\end{tabular}

CMIA/EIA: chemiluminescent microparticle immunoassay/syphilis enzyme immunoassay; RPR: rapid plasma reagin; TPHA/TP-PA: Treponema pallidum hemagglutination assay/Treponema pallidum particle agglutination assay.
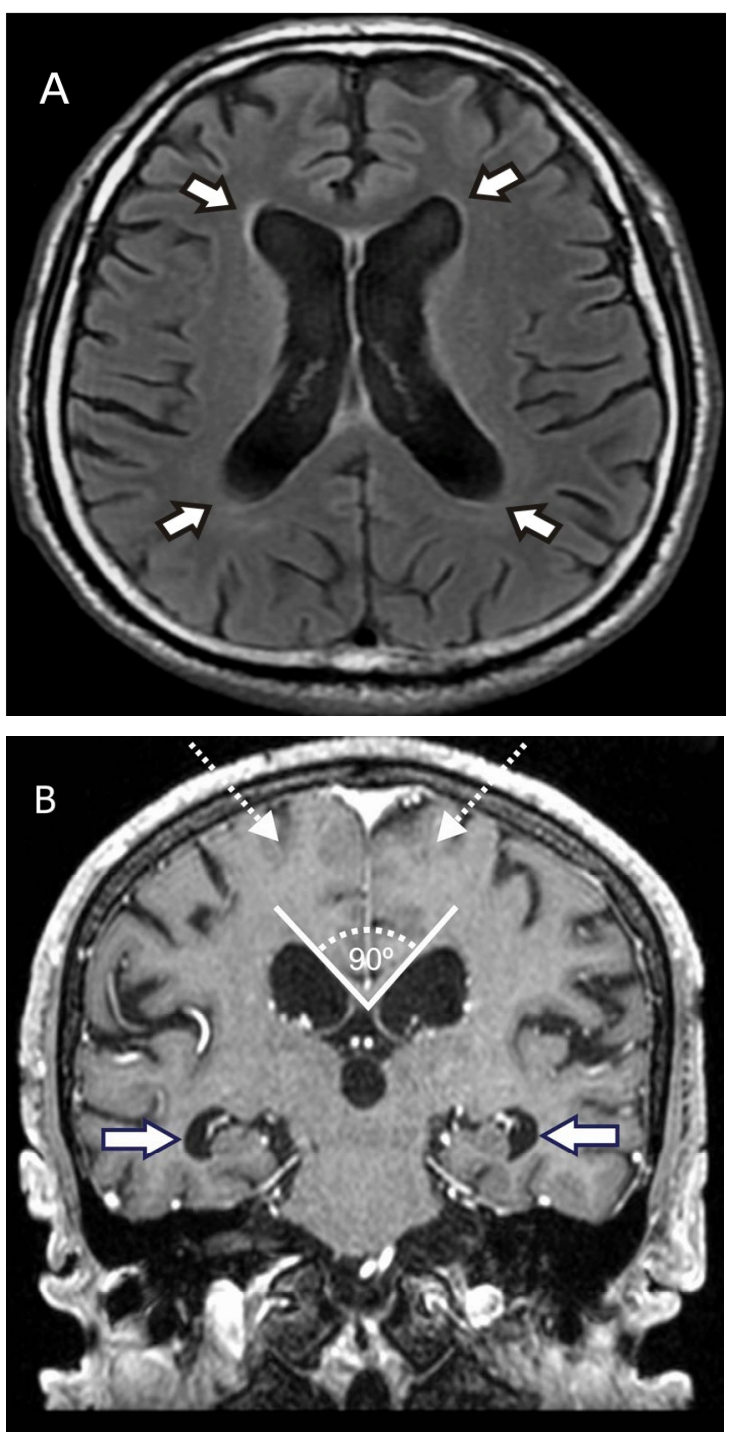

Figure 1. Brain MRI scan. (A) Axial FLAIR (Fluid Attenuated Inversion Recovery) sequence showing dilation of the ventricular system out of proportion to the degree of cerebral atrophy, and rounding of the frontal and occipital horns (arrows); (B) Coronal T2-weighted sequence showing a callosal angle of $90^{\circ}$, dilation of the temporal horns (arrows) and tight high-convexity and medial surface subarachnoid spaces (dotted arrows).
The patient was treated with crystalline penicillin (4 million units every 4 hours for 21 days) and was assessed again after three months, when his cognition and gait had improved and the urinary incontinence had resolved. A NPH scale [8] was used to measure the clinical improvement.

\section{DISCUSSION}

When syphilis is suspected, both treponemal (FTAABS, TP-PA and EIA) and non-treponemal (VDRL and RPR) tests should be carried out [1]. When the VDRL test in CSF is reactive, a diagnosis of neurosyphilis can be made. However, a nonreactive VDRL test does not exclude a diagnosis of neurosyphilis. FTA-ABS in CSF is sensitive but is not specific. However, if it is not reactive, a diagnosis of neurosyphilis can usually be excluded [1]. Our patient's serum was reactive in CMIA and RPR tests, indicating active syphilis (Table 1); his CSF findings included elevated protein levels and a nonreactive FTA-ABS test. There have been reports of rare cases in which serologic tests for syphilis in CSF have been nonreactive [1].

NPH secondary to syphilis is an atypical form of neurosyphilis with an unusual clinical presentation [3] and uncommon neuroimaging findings [4]. Pedersen et al., 1979, described a case of NPH secondary to syphilis that improved significantly with antibiotics [3]. A diagnosis of NPH is made when its triad of signs and symptoms (gait apraxia, dementia and urinary incontinence) is present together with an opening CSF pressure of less than $18 \mathrm{~cm} \mathrm{H}_{2} \mathrm{O}$ and ventricular dilation that cannot be attributed exclusively to cerebral atrophy. The neuroimaging characteristics observed in our case, which included a callosal angle of approximately $90^{\circ}$ [6], tight high-convexity and medial surface subarachnoid spaces [7] and rounding of the frontal and temporal horns, support a diagnosis of NPH. The patient's NPH score [8] was the parameter used to classify the response to treatment. The NPH scale assesses three domains (gait, cognition and sphincter control), with scores of between 1 and 5 for each item. The total score can vary from a minimum of 3 points (patient completely incapacitated) to a maximum of 15 (total independence). The result for our patient was 9/15 before treatment and 13/15 after treatment. There was an improvement of 1 point in gait and cognition and 2 points in sphincter control. The overall improvement of 4 points was considered significant.

The patient presented with the classic Hakim-Adams triad, with neuroimaging compatible with NPH. The finding of normal opening pressure excluded the possibility of hypertensive hydrocephalus. In view of the risks associated with ventriculoperitoneal shunt surgery in an elderly patient and the presence of active syphilis in the blood, we decided to administer empirical treatment for 
neurosyphilis using penicillin. The improvement in the NPH score [8] observed after administration of the antibiotic corroborated the diagnosis of atypical neurosyphilis.

\section{CONCLUSION}

Our case illustrates an atypical form of neurosyphilis (Hakim-Adams syndrome), which is considered in the literature to be a rare yet an important manifestation of this disease as it involves potentially reversible dementia. The absence of positive serology for syphilis in the CSF should not exclude a diagnosis of neurosyphilis in cases in which the clinical findings are suggestive of this infection. Physicians should bear in mind that tests can yield false-negative results and should trust their clinical impressions when these are relevant to the patient's treatment.

\section{REFERENCES}

[1] Marra, C.M. (2009) Update on neurosyphilis. Current Infectious Disease Reports, 11, 127-134. doi:10.1007/s11908-009-0019-1

[2] De Souza, R.K., Vosgerau, R.P., Sato, H.K. and Kowacs, P.A. (2013) A male patient with delirium, seizures and (limbic) encephalitis with bilateral mesial temporal lobe involvement. Arquivos de Neuro-Psiquiatria, 71, 270. doi:10.1590/0004-282X20130018

[3] Pedersen, K.K., Sorensen, P.S., Ankerhus, J. and Danielsen, U.T. (1979) Syphilitic normal pressure hydrocephalus. Acta Neurochirurgica (Wien), 48, 35-39. doi:10.1007/BF01406018

[4] Fadil, H., Gonzalez-Toledo, E., Kelley, B.J. and Kelley, R.E. (2006) Neuroimaging findings in neurosyphilis. Journal of Neuroimaging: Official Journal of the American Society of Neuroimaging, 16, 286-289.

[5] Relkin, N., Marmarou, A., Klinge, P., Bergsneider, M. and Black, P.M. (2005) Diagnosing idiopathic normalpressure hydrocephalus. Neurosurgery, 57, S4-S16.

[6] Ishii, K., Kanda, T., Harada, A., Miyamoto, N., Kawaguchi, T., Shimada, K., et al. (2008) Clinical impact of the callosal angle in the diagnosis of idiopathic normal pressure hydrocephalus. European Radiology, 18, 2678-2683. doi:10.1007/s00330-008-1044-4

[7] Hashimoto, M., Ishikawa, M., Mori, E. and Kuwana, N. (2010) Diagnosis of idiopathic normal pressure hydrocephalus is supported by MRI-based scheme: A prospective cohort study. Cerebrospinal Fluid Research, 7, 18.

[8] Sahuquillo, J., Rubio, E., Codina, A., Molins, A., Guitart, J.M., Poca, M.A., et al. (1991) Reappraisal of the intracranial pressure and cerebrospinal fluid dynamics in patients with the so-called "normal pressure hydrocephalus" syndrome. Acta Neurochirurgica (Wien), 112, 50-61. doi:10.1007/BF01402454 